\title{
LOS PECIOLOS DE LA PAPAYA COMO FUENTE DE INÓCULO DE LA ANTRACNOSIS Y SU ELIMINACIÓN COMO PRÁCTICA DE CONTROL 1
}

\author{
Alfredo Durán ${ }^{2}$ Dennis Mora², Lenín Ramírez ${ }^{2}$
}

\begin{abstract}
RESUMEN
Los peciolos de la papaya como fuente de inóculo de la antracnosis y su eliminación como práctica de control. El objetivo de este trabajo fue determinar el número de esporas de hongos que causan enfermedades en los frutos de papaya y medir la influencia que tiene la eliminación de peciolos, sobre la población de propágulos de los hongos. Los peciolos colectados durante la estación lluviosa liberaron mayor cantidad ( $82 \%$ ) de conidios de Colletotrichum sp. que los colectados durante la estación seca. En los peciolos de la estación lluviosa, se dio una reducción en la cantidad de conidios liberados $(77 \%)$ de la primera a la segunda semana después de la colecta. Tanto para el periodo seco, como para el lluvioso, después de cuatro semanas, los peciolos colectados continuaron liberando conidios en bajas cantidades. Las mayores cantidades de ascosporas de Glomerella sp. se capturaron durante los meses de abril a junio. La captura de conidios de Colletotrichum sp. con y sin eliminación de peciolos durante los meses de abril a junio presentó valores que aumentaron, hasta alcanzar sus máximos, entre setiembre y octubre. Los valores de captura de propágulos en los lotes con y sin prácticas de eliminación de peciolos fueron similares para Glomerella sp. La eliminación de peciolos produjo una reducción en la captura de esporas, para los hongos Colletotrichum sp., Helminthosporium sp. y Fusarium sp., mientras que para los hongos Curvularia sp. y Alternaria sp. la eliminación no mostró ningún efecto. La estación lluviosa favoreció la captura de esporas de los hongos Helminthosporium sp., Curvularia sp. y Fusarium sp., cuando se comparó con la estación seca, mientras que se capturó un mayor número de esporas del hongo Alternaria sp. durante la estación seca.
\end{abstract}

\begin{abstract}
Stems of papaya as a source for anthracnose and their elimination as a control practice. The objective of this study was to determine the number of spores of fungi that cause diseases in the fruits of papaya and to measure the influence that petiole elimination could have on the population of disease propagating agents. The stems collected during the rainy season released a higher number (82\%) of Colletotrichum sp. conidia than those collected during the dry season. In the stems collected during the rainy season a reduction was observed in the amount of conidia released (77\%), between the first and the second week after collection. The stems collected during the dry and rainy season released a small amount of conidia after four weeks of collection. The higher numbers of Glomerella sp. ascospores captured during April to June. The capture of Colletotrichum sp. conidia with and without elimination of stems showed values that increased from April to June with a maximum between September and October. The numbers of disease propagating agents captured in the plots with and without stem elimination were similar for Glomerella sp. The elimination of stems caused a reduction in the number of spores captured in Colletotrichum sp., Helminthosporium sp. and Fusarium sp. fungi. Stem elimination caused no effect on Curvularia sp. and Alternaria sp. fungi. The rainy season was more favorable for the collection of spores of Helminthosporium sp., Curvularia sp. and Fusarium sp., compared to that from the dry season. For Alternaria sp. a higher number of spore was collected during the dry season.
\end{abstract}

\section{INTRODUCCIÓN}

La antracnosis es la enfermedad más importante de la fruta de papaya en Costa Rica. En 1981, Arauz y
Mora informaron sobre los niveles que alcanza y de sus efectos en la etapa postcosecha sobre la producción del país. Durán y Mora en 1987 confirmaron lo anterior, en estudios en donde detallan y aclaran la sintoma-

\footnotetext{
1 Proyecto inscrito en la Estación Experimental Fabio Baudrit M., Vicerrectoría de Investigación, Universidad de Costa Rica.

2 Estación Experimental Fabio Baudrit y Laboratorio de Fitopatología, Escuela de Fitotecnia, Universidad de Costa Rica.
} 
tología, aportan datos de incidencia y severidad en las principales zonas productoras del país y analizan la relación entre éstas y la precipitación.

A nivel mundial, la antracnosis también ha sido reportada, desde hace muchos años, como la enfermedad de mayor importancia en Hawaii (Hunter y Buddenhagen, 1972), Brasil (Bolkan, 1976), Australia (Simmonds, 1937) y otros lugares, según lo describen múltiples autores como Brum, 1952; Frossard,1969; Raabe y Holtzman, 1964.

$\mathrm{La}$ antracnosis de la papaya es producida por $\mathrm{Co}$ lletotrichum gloesporioides, un hongo de la familia de los Melanconiales, se reproduce mediante conidios producidos en acérvulos, se diseminan a través, principalmente, de la combinación de agua y viento (Agrios 1995).

La antracnosis, además de diseminarse a través de los conidios de Colletotrichum sp., puede llegar a los frutos a través de ascósporas de su forma perfecta, Glomerella sp. al ser liberadas por los peritecios y se diseminan principalmente a través de viento. Esta situación fue descrita por Hunter y Buddenhagen en Hawaii (1972) y por Durán y Mora (1988b) en Costa Rica, quienes además indican, la dificultad que existe para relacionar los niveles de incidencia y severidad de la enfermedad, con los niveles de lluvia en una plantación.

El hongo además de atacar frutos, es capaz de causar daños en flores y pecíolos según lo ha descrito en Costa Rica, Durán y Mora (1987).

Los niveles de incidencia y severidad causados por la enfermedad son usualmente tan altos, que su combate se vuelve imprescindible para obtener producciones rentables y aceptables por el mercado. Sin embargo, por ser una fruta de consumo fresco, existen grandes limitaciones para el uso de fungicidas, razón por la cual cobran mucha importancia otras formas de combate integrado, como la sanidad vegetal, para manejar las fuentes de inóculo.

La posibilidad de que otras plantas, o los pecíolos de las hojas viejas depositados en grandes cantidades sobre el suelo, se conviertan en fuente de inóculo, adquiere mucha importancia, desde el punto de vista de la necesidad de establecer sistemas de sanidad vegetal, para combatir o prevenir la enfermedad.

Castro, Wang y Arauz (1996) y Castro, Arauz y Wang (1996) al respecto, informan de estudios en este sentido, indicando que los pecíolos colgantes de la planta, podrían constituirse en una fuente importante de conidios de Colletotrichum sp. capaces de infectar la fruta en el campo, al demostrar, mediante inoculaciones, la infectividad de los conidios de los peciolos sobre el fruto. Sin embargo, la práctica de la remoción quincenal de peciolos, no provocó diferencias significativas en la incidencia y severidad de la enfermedad, respecto al testigo sin remoción.

Ureña y Arauz (1994) indican que la práctica de la remoción de peciolos de papaya caídos al suelo, junto con la aplicación de fungicidas, provocó una reducción en la cantidad de esporas capturadas en trampas, sin embargo, esta reducción fue muy baja, por lo que concluyeron que otras fuentes de inóculo, como los peciolos senescentes podrían tener una gran influencia en la diseminación del hongo a nivel del fruto.

Como los niveles de incidencia de la antracnosis en el fruto de la papaya generalmente son muy altos y los frutos enfermos no desarrollan síntomas que esporulen bajo condiciones de campo, se planteó la duda de si los pecíolos no son señalados como fuente importante de inóculo primario, debería existir, entonces, otra fuente importante de propágulos de la enfermedad.

Dado que es remota la posibilidad de que otros cultivos, incluso los hospederos alternos (Ramírez et al, 1996), sean fuente de inóculo importante en plantaciones grandes de papaya y que aún no se han encontrado malezas hospedantes del hongo en Costa Rica, se decidió realizar más estudios, pero de largo plazo, para determinar los valores de captura de esporas liberadas a partir de pecíolos de papaya y la influencia que podrían tener las prácticas de sanidad vegetal, sobre la reducción de la diseminación de los propágulos que sirven de inóculo para la antracnosis de la fruta.

De igual forma, se realizaron estudios con el fin de determinar durante cuánto tiempo, un pecíolo es capaz de actuar como fuente de inóculo de la antracnosis.

\section{MATERIALES Y MÉTODOS}

\section{Evaluación del periodo de actividad de los pecíolos de papaya como fuente de inóculo de la antracnosis de la fruta y del potencial de producción de inóculo.}

Durante el periodo comprendido entre el 18 de febrero y el 30 de marzo de 1996 se realizó la primera etapa, para evaluar el periodo de la estación seca y entre el 5 de setiembre y el 10 de octubre del mismo año se realizó la segunda etapa del experimento, para investigar el periodo correspondiente a la estación lluviosa. 
Las evaluaciones se desarrollaron en una plantación comercial de 50 ha de papaya, de la variedad hawaiiana, Solo Sunrise, ubicada en Paquera, provincia de Puntarenas, zona que corresponde a un bosque tropical lluvioso según la clasificación de Holdridge y el mapa de zonas de vida de Tosi ( 1969).

El procedimiento consistió en recoger al menos 20 pecíolos senescentes, pero aún colgantes, de árboles de papaya. Los pecíolos se tomaron de diferentes sectores de la plantación y se introdujeron en una bolsa de nylon. Se prepararon cuatro bolsas de iguales características, las cuales se colgaron de igual número de árboles en el centro de la plantación. Se dejaron expuestos a la acción del ambiente, tanto de la lluvia como del viento, por periodos variables de tiempo, según el tratamiento. La primera bolsa se retiró a la semana de estar a la intemperie y las bolsas restantes se fueron retirando una a una, a la segunda, tercera y cuarta semana de iniciado el experimento, esto se realizó de igual forma para la época de verano e invierno.

Al día siguiente que se retiró del campo cada bolsa, se procedió a extraer las esporas del hongo Colletotrichum gloeosporioides, presentes en los pecíolos. Los pecíolos se sacaron de la bolsa; se escogieron diez que tuvieran presencia de acérvulos de Colletotrichum sp. De cada uno se seleccionó una sección de $20 \mathrm{~cm}$ de largo en donde se observó mayor concentración de acérvulos. Cada sección de pecíolo se colocó por separado en un mortero con $50 \mathrm{ml}$ de agua destilada y luego se trituró el tejido por un periodo aproximado de tres minutos. Luego, se procedió a filtrar cada una de las 10 suspensiones de esporas, primero usando una maya de gasa doble y posteriormente, a través de un filtro milipor 678 , con la ayuda de una bomba de vacío.

Una vez listas las suspensiones de esporas (una para cada trozo de pecíolo), se procedió a contar las esporas de Colletotrichum sp. presentes, utilizando un hematocímetro. Se realizaron cuatro conteos para cada solución, los cuales posteriormente se promediaron, para obtener la cantidad de esporas por mililitro extraídas de cada pecíolo.

\section{Evaluación de la influencia de la eliminación de peciolos sobre la captura de conidios y ascósporas de antracnosis a nivel del fruto.}

Se efectuó, a la misma altura de las frutas, un trampeo de esporas de Colletotrichum sp. y Glomerella sp., causantes de la antracnosis de la papaya, en la misma plantación en que se realizó el estudio anterior.
El objetivo de los experimentos fue determinar la importancia de los pecíolos de papaya senescentes, como diseminadores de conidios o ascósporas de antracnosis, a la altura de las frutas y evaluar el efecto de la práctica de sanidad vegetal, mediante su recolección semanal, sobre los niveles de captura de ambos tipos de propágulos, tanto bajo condiciones de estación seca, como lluviosa.

En una plantación de 50 hectáreas de extensión, en la cual se aplicaba la práctica de recolección y eliminación constante de los peciolos y en el centro de una sección de 20 hectáreas, se marcaron dos lotes de $625 \mathrm{~m}^{2}$ cada uno. En un lote se permitió, desde la siembra, la acumulación de hojas y pecíolos secos de papaya y en el otro, ubicado a $100 \mathrm{~m}$ de distancia, se recolectaron y extrajeron los desechos del cultivo desde que se transplantó, con una frecuencia de dos veces por semana.

En cada lote se ubicaron 20 trampas de vidrio de ocho por 2,5 cm (portaobjetos), cubiertas por ambos lados con cinta adhesiva impermeable y sujetadas a los árboles de papaya, justo debajo de la fruta de más edad. De las 20 trampas, se ubicaron 10 de ellas en forma perpendicular a la superficie del suelo y las otras 10 en forma paralela. Cada semana se cambiaron las trampas con el fin de hacer el conteo de esporas, además se ajustó la altura de las trampas, para mantenerlas siempre debajo de la fruta de mayor edad.

El trampeo se inició la primera semana de abril, cuando la plantación tenía cuatro meses de edad y concluyó en la última semana de octubre, cuando tenía cuatro meses de producción.

En cada una de las trampas retiradas cada semana, se hizo el conteo, mediante una cuidadosa observación al microscopio de toda el área de las dos caras del vidrio, anotando el número total de conidios de Colletotrichum sp. y de ascósporas de Glomerella sp. capturadas por trampa.

En cada trampeo semanal se hizo un total de 20 lecturas por lote, con y sin práctica de sanidad vegetal, para un total de 560 lecturas por lote durante el experimento.

A la vez que se hicieron lecturas del número de propágulos diseminadores de la antracnosis del fruto, se contó el número de conidios presentes en cada trampa de otros tipos de hongos de importancia menor en el cultivo, como algunos productores de enfermedades de la flor y de las hojas.

Para llevar un control de la precipitación durante el transcurso de las observaciones, se instaló a 100 me- 
tros del borde de la plantación un pluviómetro, las lecturas se hicieron diariamente.

\section{RESULTADOS Y DISCUSIÓN}

\section{Evaluación del periodo de actividad de los pecíolos de papaya como fuente de inóculo de la antracnosis del fruto y del potencial de producción de inóculo.}

En el Cuadro 1 se presentan los datos de conteo de conidios a partir de la extracción realizada en los pecíolos. Estos resultados indican que los pecíolos colectados durante la estación lluviosa liberan mayor cantidad $(80,2 \%)$ de conidios de Colletotrichum sp. que los pecíolos colectados durante la estación seca. Lo anterior puede explicarse desde el punto de vista de que los conidios dentro de los acérvulos necesitan de la combinación de agua y lluvia para ser liberados y diseminados, tal y como lo indica Agrios (1995) y en el hecho de que, por ser menor la liberación durante el verano, es menor también el inóculo primario disponible para completar el proceso de infección en pecíolos verdes, aparte de que la condición de baja humedad relativa, propia de la estación seca, no favorece el desarrollo de nuevas infecciones en los peciolos.

Debe considerarse que en la región en donde se realizó la investigación, la estación seca inicia a principios de noviembre y las lluvias se regularizan hasta finales de junio, razón por la cual se da una importante producción de hojas nuevas en la planta durante esta época, cuyos pecíolos han sido sometidos a una menor presión de inóculo de conidios durante su desarrollo. De igual forma, los frutos que cumplan su período de 16 semanas de desarrollo durante los meses de la estación seca, también estarán sometidos a menor presión de inóculo de antracnosis producida por conidios.

En los pecíolos de la estación lluviosa, se da una reducción significativa en la cantidad de conidios colectados $(77 \%)$ entre la primera y la segunda semana de se- parados de la planta. Lo anterior tiene su explicación en el hecho de que justamente por ser mayor la liberación de conidios en la estación lluviosa, durante este período los acérvulos rápidamente descargan su contenido.

Por otra parte, en la estación lluviosa la velocidad de descomposición de los pecíolos es más rápida, razón por la cual la reducción en la cantidad de conidios liberados conforme se descompone el pecíolo en el campo, alcanza valores más altos en la estación lluviosa que en la estación seca. Posterior a la segunda semana, la reducción en la liberación de conidios continúa, pero a niveles inferiores, tanto cuando se analizan los datos de la estación lluviosa, como los de la estación seca.

Con relación al tiempo en que el pecíolo se mantiene activo como fuente de inóculo, se obtuvo que, para los dos periodos, seco y lluvioso, después de cuatro semanas, los pecíolos continúan liberando conidios, aunque en cantidades muy bajas, comparadas con los valores máximos que se observaron durante la primera semana de liberación.

A juicio de los autores y con base en experiencias de manejo comercial de la antracnosis conducidas durante dos años en la plantación en donde se realizaron estas investigaciones, los resultados obtenidos indican, la necesidad de analizar la utilidad de las prácticas de sanidad vegetal, mediante la recolección de los pecíolos senescentes en el campo.

Para ello, se considera necesario cuantificar la cantidad de conidios que llega a nivel de la fruta, tanto en estación seca como lluviosa. Será necesario además, evaluar la periodicidad de una práctica de sanidad vegetal, considerando la reducción de las cantidades liberadas, conforme avanza el tiempo de descomposición del pecíolo, así como la pertinencia de realizar esta labor de limpieza de la plantación durante el verano, dadas las cantidades reducidas en la liberación de conidios, que se observaron en comparación con la estación lluviosa.

Cuadro 1. Datos de la liberación mínima y máxima de esporas de Colletotrichum gloeosporioides en los pecíolos senescentes de papaya según tratamientos. Paquera, Costa Rica, 1996.

\begin{tabular}{|c|c|c|c|c|c|c|c|c|}
\hline & \multicolumn{8}{|c|}{ Semanas a la intemperie } \\
\hline & \multicolumn{2}{|c|}{1} & \multicolumn{2}{|r|}{2} & \multicolumn{2}{|c|}{3} & \multicolumn{2}{|l|}{4} \\
\hline & $\begin{array}{c}\text { Estación } \\
\text { seca }\end{array}$ & $\begin{array}{l}\text { Estación } \\
\text { lluviosa }\end{array}$ & $\begin{array}{c}\text { Estación } \\
\text { seca }\end{array}$ & $\begin{array}{l}\text { Estación } \\
\text { lluviosa }\end{array}$ & $\begin{array}{c}\text { Estación } \\
\text { seca }\end{array}$ & $\begin{array}{l}\text { Estación } \\
\text { lluviosa }\end{array}$ & $\begin{array}{c}\text { Estación } \\
\text { seca }\end{array}$ & $\begin{array}{l}\text { Estación } \\
\text { lluviosa }\end{array}$ \\
\hline Promedio* & 354.500 & 2.355 .000 & 187.700 & 535.750 & 60.750 & 194.500 & 34.750 & 142.750 \\
\hline Lib. Máxima & 635.000 & 4.787 .500 & 405.000 & 1.160 .000 & 127.500 & 375.000 & 115.000 & 267.500 \\
\hline Lib. Mínima & 150.000 & 980.000 & 50.000 & 187.500 & 15.000 & 62.500 & 7.500 & 35.000 \\
\hline
\end{tabular}

* Promedio de esporas por cc en 10 pecíolos y cuatro lecturas a cada uno. 
Evaluación de la influencia de la sanidad vegetal sobre la captura de conidios y ascósporas de antracnosis a nivel del fruto

Los datos de la precipitación durante el período de observaciones se presenta en la Figura 1. La precipitación puede considerarse normal para la zona en donde está ubicada la plantación.

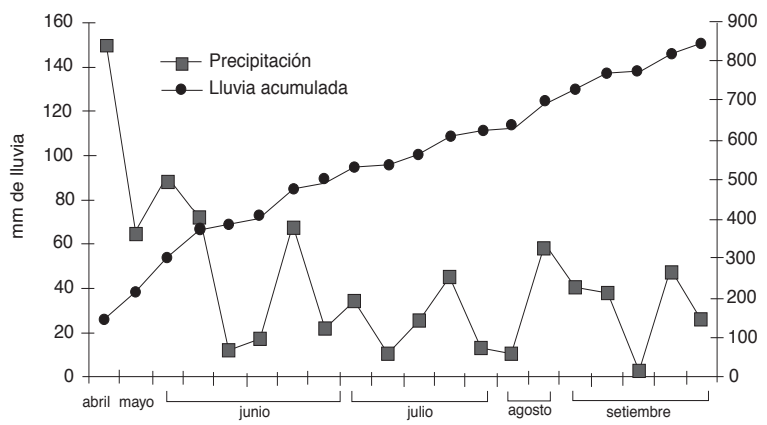

Figura 1. Precipitación y lluvia acumulada durante el período del experimento, Paquera, 1996.

El análisis general de los datos de la Figura 2 indica que las mayores cantidades de ascósporas de Glomerella sp. se capturaron durante los meses secos, de abril a junio, con valores acumulados para ambos lotes de 20 esporas, mientras que en los meses lluviosos, hacia el final del experimento, se presentaron valores acumulados de captura de una espora.

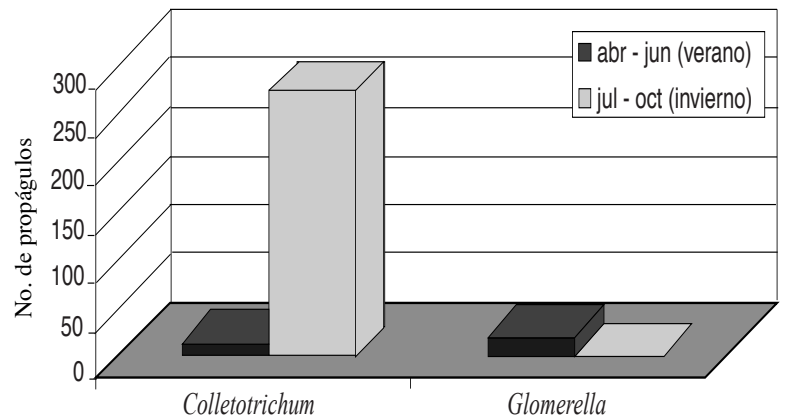

Figura 2. Captura de propágulos diseminadores de la antracnosis de la papaya, durante el período seco y lluvioso, Paquera, 1996.

La captura de conidios de Colletotrichum sp. en ambos lotes, durante los meses de abril a junio, presentó valores acumulados de 13 conidios, que aumentaron, con la regularización de las lluvias, hasta alcanzar sus valores máximos, entre setiembre y octubre, con valores acumulados de 273 conidios capturados. Lo anterior coincide con la relación presentada por Agrios (1995) entre el tipo de estructura de diseminación, la estructura que lo contiene y el agente diseminador de cada uno, puesto que la lluvia se encarga de promover la liberación de los conidios a partir de los acérvulos de los pecíolos y el viento de diseminar las ascósporas, a partir también de los pecíolos. Por otra parte, también es más frecuente que se presente la forma perfecta del hongo cuando las condiciones le son adversas, como en la estación seca.

Estos datos explican la razón por la que la enfermedad es capaz de diseminarse aún en condiciones de estación seca, llegando a producir infecciones sobre los frutos, y además, por qué la fruta producida en el verano, en general, presenta valores de incidencia y severidad de antracnosis inferiores, que la fruta de estación lluviosa, como lo señalaron Durán y Mora en 1987.

Con referencia al efecto sobre la antracnosis, de la práctica de sanidad vegetal mediante la remoción de peciolos del papayal (Figuras 3 y 4), se encontró que la tendencia general de la captura, tanto de esporas de Glomerella sp., como de conidios de Colletotrichum sp. es similar entre los lotes, con y sin práctica de sanidad vegetal, puesto que la mayor captura de ascósporas se da en el periodo seco, para ambos lotes, mientras que la mayor captura de conidios se da en el periodo lluvioso, aunque con diferencias en la cantidad capturada en los lotes con y sin sanidad.

Cuando se analizan los valores de captura de propágulos en los lotes con y sin prácticas de sanidad vegetal (Figura 5), se observa que son prácticamente iguales para las esporas de Glomerella sp., ya que se capturaron 10 y 11 esporas respectivamente. Para los conidios de Colletotrichum sp. la situación es diferente, puesto que se capturaron 98 y 188 conidios respectivamente, lo que representa una reducción del 49,1\% como efecto de la sanidad vegetal, sobre el lote en donde no se realizó esta práctica y se permitió la acumulación de residuos del cultivo sobre el suelo.

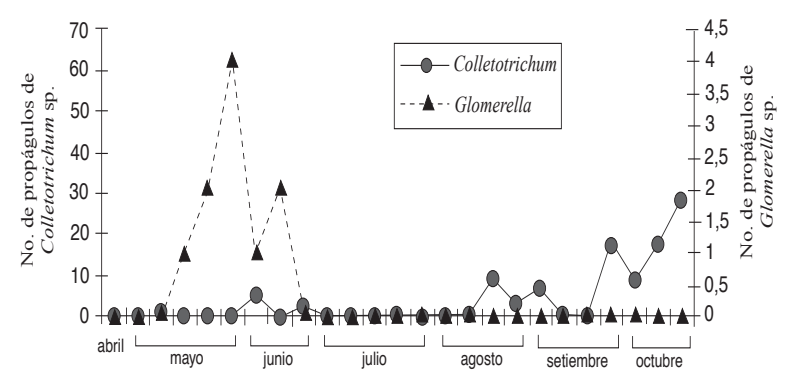

Figura 3. Captura de propágulos diseminadores de la antracnosis de la papaya en la parcela con prácticas sanitarias. Paquera, 1996. 


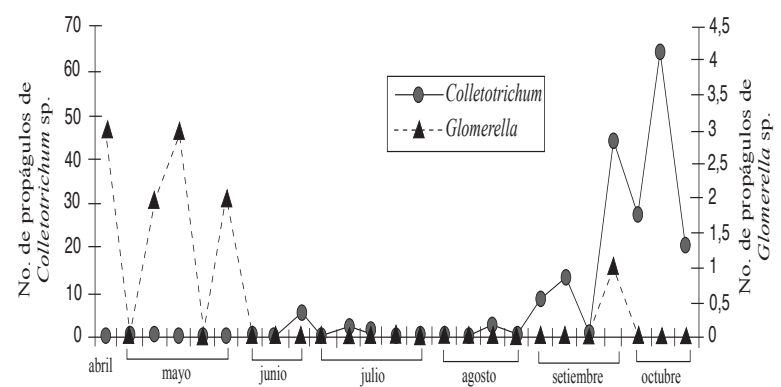

Figura 4. Captura de propágulos diseminadores de la antracnosis de la papaya en la parcela sin prácticas sanitarias. Paquera, 1996

Los datos anteriores confirman la estrecha relación que existe entre la diseminación de la enfermedad y la presencia de lluvia y viento como en la estación lluviosa, o la presencia de sólo viento como en la estación seca. Esto encuentra su explicación en la mecánica de liberación de los propágulos a partir de los acérvulos, en el caso de los conidios de Colletotrichum sp., o de los peritecios, en el caso de las ascósporas de Glomerella sp., según lo explica Agrios (1995). Esto mismo explica la razón de por qué se observa la presencia de la enfermedad, con niveles bajos de incidencia, en frutas que se han desarrollado en ausencia de lluvia y además la errática relación que observaron Durán y Mora, 1988b, entre la precipitación y los valores de incidencia y severidad de la antracnosis en la fruta.

Desde el punto de vista del manejo de la enfermedad, los datos obtenidos y analizados indican la necesidad de mantener medidas para su erradicación durante la estación seca, en la planta de empaque, cuando se trata de fruta para la exportación y la importancia que tiene, durante la estación lluviosa, la práctica de sanidad vegetal, mediante la recolección de los pecíolos, puesto que una reducción de casi el $50 \%$ de las esporas que llegan a la fruta, puede contribuir a disminuir el uso de los fungicidas en el campo al reducir la incidencia de la enfermedad y aumentar la efectividad de las prácticas poscosecha, tales como el tratamiento de agua caliente, la

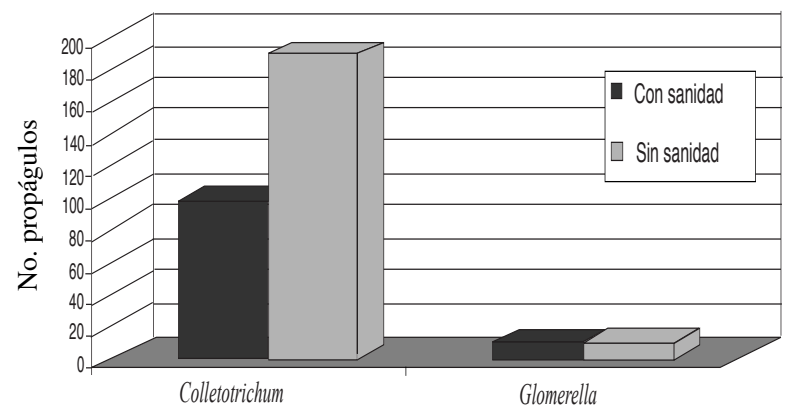

Figura 5. Cantidad de propágulos diseminadores de la antracnosis de la papaya en parcelas con y sin prácticas de sanidad. Paquera, 1996. inmersión en soluciones fungicidas y el almacenamiento en frío de la fruta que se aplican en planta de empaque.

A pesar de que la práctica de recolección de peciolos fue parcialmente efectiva en eliminar el inóculo, los resultados obtenidos fueron diferentes a los generados por Castro et al., 1996 y Ureña y Arauz, 1994, en donde la práctica de recolección regular de los peciolos no mostró reducciones significativas en la población de conidios, ni en el nivel de daño de la enfermedad. En este caso, es probable que haya influido el hecho de que la plantación tenía como práctica regular, la recolección y eliminación de peciolos, y lo que se hizo fue dejar en el área de experimentación, un lote en donde se acumularon los peciolos. Es decir, por su manejo, los niveles de inóculo totales de la plantación, eran menores que los que se podían encontrar en una plantación comercial sin manejo de estos residuos, lo cual permitió detectar las diferencias. Adicionalmente, Ureña y Arauz, 1994, sólo eliminaron los peciolos depositados en el suelo, permitiendo la liberación de conidios en los peciolos senescentes aún colgantes, e indican que la práctica de recolección la realizaron quincenalmente. Al contrario en este ensayo, se recogieron los pecíolos colgantes y depositados en el suelo y la recolección se hizo con mucho mayor frecuencia, puesto que es durante la primera semana de senescencia de los pecíolos (Cuadro 1), cuando ocurre la mayor liberación de conidios de Colletotrichum sp.

\section{Efecto de la eliminación de peciolos sobre el trampeo de esporas de otros hongos presentes en el papayal.}

Con relación al efecto de la práctica de eliminación de peciolos, sobre otros hongos presentes en la plantación de papaya, se tuvo que la práctica de sanidad vegetal produjo una reducción en la captura de esporas, al comparar el lote con sanidad y el lote sin esta práctica, para el hongo Helminthosporium $s p$ con capturas de 0 y 145 esporas respectivamente. También se encontró un leve efecto para el hongo Fusarium sp. con valores de cero y 18 esporas respectivamente (Figura 6). Para los

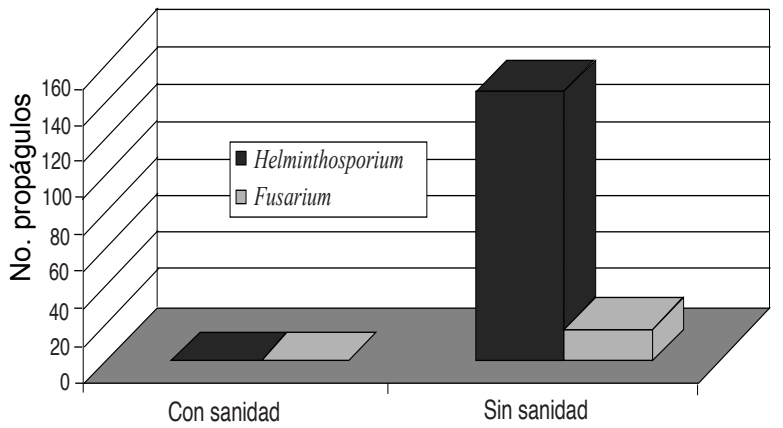

Figura 6. Cantidad de propágulos de hongos moniliales en parcelas con y sin recolección de peciolos de papaya. Paquera, 1996. 
hongos Curvularia sp. y Alternaria sp., la práctica de sanidad vegetal no produjo ningún efecto (Figura 7), puesto que en los lotes con recolección de peciolos, los valores fueron más altos, en comparación con los lotes con desechos, tanto para el hongo Curvularia sp. con valores de 183 y 42, como para el hongo Alternaria sp. con valores de 2592 y 1729 respectivamente.

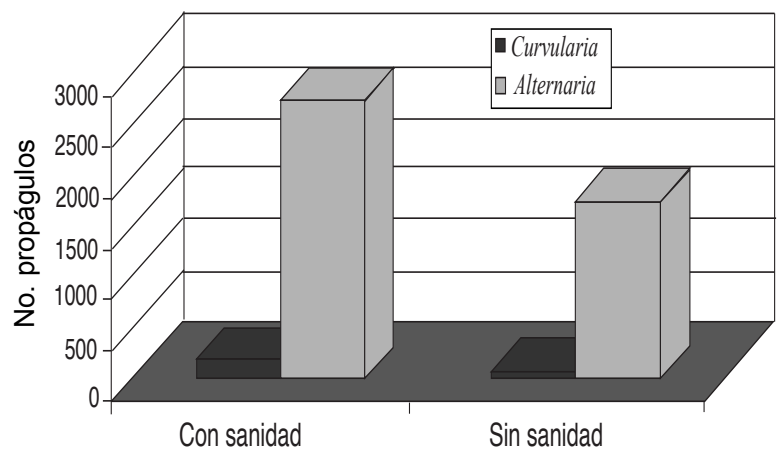

Figura 7. Cantidad de propágulos de hongos moniliales en parcelas con y sin recolección de peciolos de papaya. Paquera, 1996.

La condición lluviosa favoreció la captura de esporas de los hongos Helminthosporium sp.; Curvularia sp. y Fusarium sp. (Figuras 8 y 9), cuando se comparó con la condición de estación seca. Por el contrario, se presentó mayor captura de esporas del hongo Alternaria sp. durante el verano, con valores de 2606 esporas capturadas en la estación seca, contra 1715 en época lluviosa. Para los otros hongos, los valores de captura, al comparar la estación lluviosa contra la estación seca, fueron de 152 y 73 esporas de Curvularia sp.; 78 y 67 esporas de Helminthosporium sp. y 18 y 0 esporas de Fusarium sp., respectivamente.

De los hongos mencionados, únicamente Fusarium $s p$ es un patógeno importante en flores y frutos de pa-

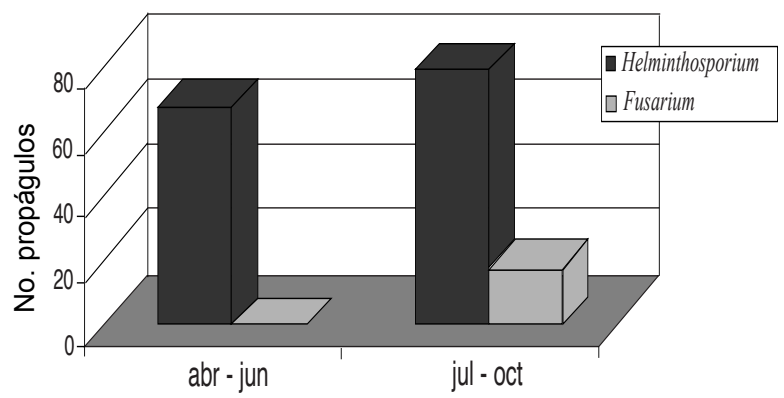

Figura 8. Cantidad de propágulos de hongos moniliales capturados a nivel del fruto según la época del año. Paquera, 1996.

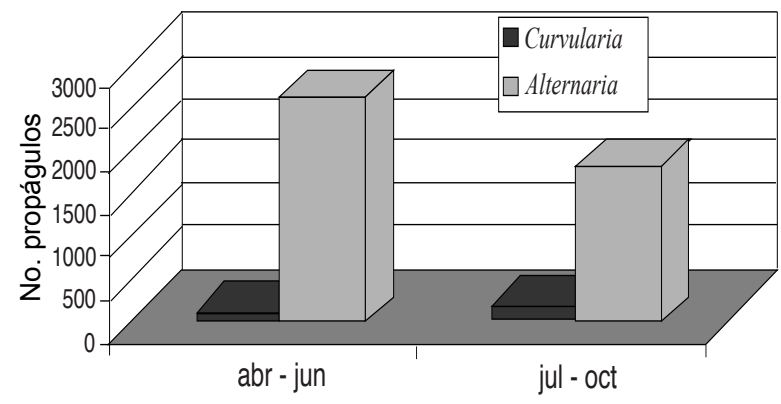

Figura 9. Cantidad de propágulos de hongos moniliales capturados a nivel del fruto según la época del año. Paquera, 1996.

paya pequeños, en donde causa su necrosis y posterior caída, alcanzando incidencias de hasta el 80\%, según informan Durán y Mora en 1982a.

Los hongos Curvularia sp., Helminthosporium sp. y Alternaria sp., aunque no son patógenos importantes de fruta, sí han sido reportados causando infecciones en el fruto en India (Srivastava, 1964 y Prassad y Verma, 1976 ). Además, causan infecciones en hojas viejas, sobre todo en condiciones de fuertes y prolongadas lluvias con viento, provocando su amarillamiento y posterior caída, lo cual tiene un efecto indirecto sobre la fruta, puesto que la expone al sol. Por otro lado, Durán y Mora, 1988a, han informado para Costa Rica, de la infección por Curvularia sp. en frutos desarrollados que han sufrido heridas.

Curvularia sp y Fusarium sp. ya habían sido reportados por Durán y Mora en 1982b, como dos de los hongos que se encuentran como patógenos de los pecíolos senescentes, produciendo estructuras de diseminación.

\section{LITERATURA CITADA}

AGRIOS, G. 1995 . Fitopatología. Editorial Limusa, segunda edición en español, traducción hecha por Manuel Guzmán Ortíz, México, pp. 337-344.

ARAUZ, L.F.; MORA, D. 1983. Evaluación preliminar de los problemas postcosecha en seis frutas tropicales de Costa Rica. Agronomía Costarricense 7 (1/2): 43-53.

BOLKAN, H. A. 1976. Fungi associated with pre and post harvest fruit rots of papaya and their control in Central Brazil. Plant Dis. Rep., 60 (7): 605-609.

BRUM, J. 1952. Sur quelques parasites responsables des anthracnoses de la papaye. Fruits d'utre Mer, 7 (6): 271-272. 
CASTRO, O.; ARAUZ, F.; WANG, A. 1996. Importancia de los peciolos de papaya como fuente de inóculo de la antracnosis del fruto de la papaya. In: Memorias X Congreso Nacional Agronómico y III Congreso de Fitopatología. San José, Costa Rica, p. 123.

CASTRO, O.; WANG, A.; ARAUZ, F. 1996. Efecto de la remoción de peciolos y la aplicación de fungicidas en el combate de antracnosis (Colletrotrichum gloeosporioides) en papaya. . In: Memorias X Congreso Nacional Agronómico y III Congreso de Fitopatología. San José, Costa Rica, p. 127.

DURAN, A.; MORA, D. 1982a. Etiología de la momificación de las flores y frutos de la papaya en Guanacaste, Costa Rica. In: Memorias V Congreso Agronómico Nacional, San José, Costa Rica, p. 63-64.

DURAN, A.; MORA, D. 1982b. Los peciolos de papaya como posible fuente de inóculo de las enfermedades postcosecha del fruto. In: Memorias V Congreso Agronómico Nacional, San José, Costa Rica, pp. 65-66.

DURAN, A.; MORA, D. 1987. Variabilidad de la sintomatología de la antracnosis (Colletrotrichum sp.) en papaya, durante el periodo poscosecha en Costa Rica. Agronomía Costarricense 11 (2): 155-161.

DURAN, A.; MORA, D. 1988a. Diagnóstico de las enfermedades postcosecha de la papaya en Costa Rica: I. Pruebas de patogenicidad. Agronomía Costarricense 12 (1): 1-6.

DURAN, A.; MORA, D. 1988b. Diagnóstico de las enfermedades postcosecha de la papaya en Costa Rica: II. Cuantificación y epidemiología de las enfermedades del fruto. Agronomía Costarricense 12 (1): 7-18.
FROSSARD, P. 1969. Maladies des fruits. Fruits d'utre Mer, 24 (11): 473-482.

HUNTER, J. E.; BUDDENHAGEN, I. W. 1972. Incidence, epidemiology and control of fruit diseases of papaya in Hawaii. Trop. Agric. (Trinidad), 49 (1): 61-71.

PRASSAD, J. S; VERMA, A. B., 1976. Investigations on the diseases of papaya. IV. Post-infection changes in ascorbic acid content. Indian Phytopatology, 29 (1): 84-85.

RAABE, R. D.; HOLTZMAN, O. V. 1964. Studies on the control of papaya anthracnose. Hawaii Farm Sc., 13 (4): $1-2$

RAMIREZ, L.; DURAN, A.; MORA, D. 1996. Estudios iniciales sobre los diferentes hospederos alternos del hongo Colletotrichum sp., causante de la antracnosis de la papaya en Costa Rica. In: Memoria X Congreso Agronómico Nacional, San José, Costa Rica, p. 95.

SIMMONDS, J. H. 1937. Diseases of the papaya. Queensland Agriculture Journal. 48: 544-552.

SRIVASTAVA, M. P. 1964. Studies on fungal diseases of some tropical fruits. I. A list of the fungi isolated from fruits and trees. Phytopath. Zeitschr., 50: 250-261.

TOSI, J. A. 1969. República de Costa Rica: Mapa Ecológico según la clasificación de zonas de vida del mundo, de L. R. Holdridge., Centro Científico Tropical, San José, Costa Rica, Escala 1: 750000

UREÑA, A.; ARAUZ, F. 1994. Dinámica poblacional de Colletotrichum gloeosporioides en papaya (Carica papaya), cultivada bajo diferentes sistemas de manejo. In. Memorias V Congreso Internacional de Manejo Integrado de Plagas. San José, Costa Rica, p. 73. 